\section{Therapeutic strategy for removal of a large dental prosthesis with a sharp clasp, embedded in the esophagus}
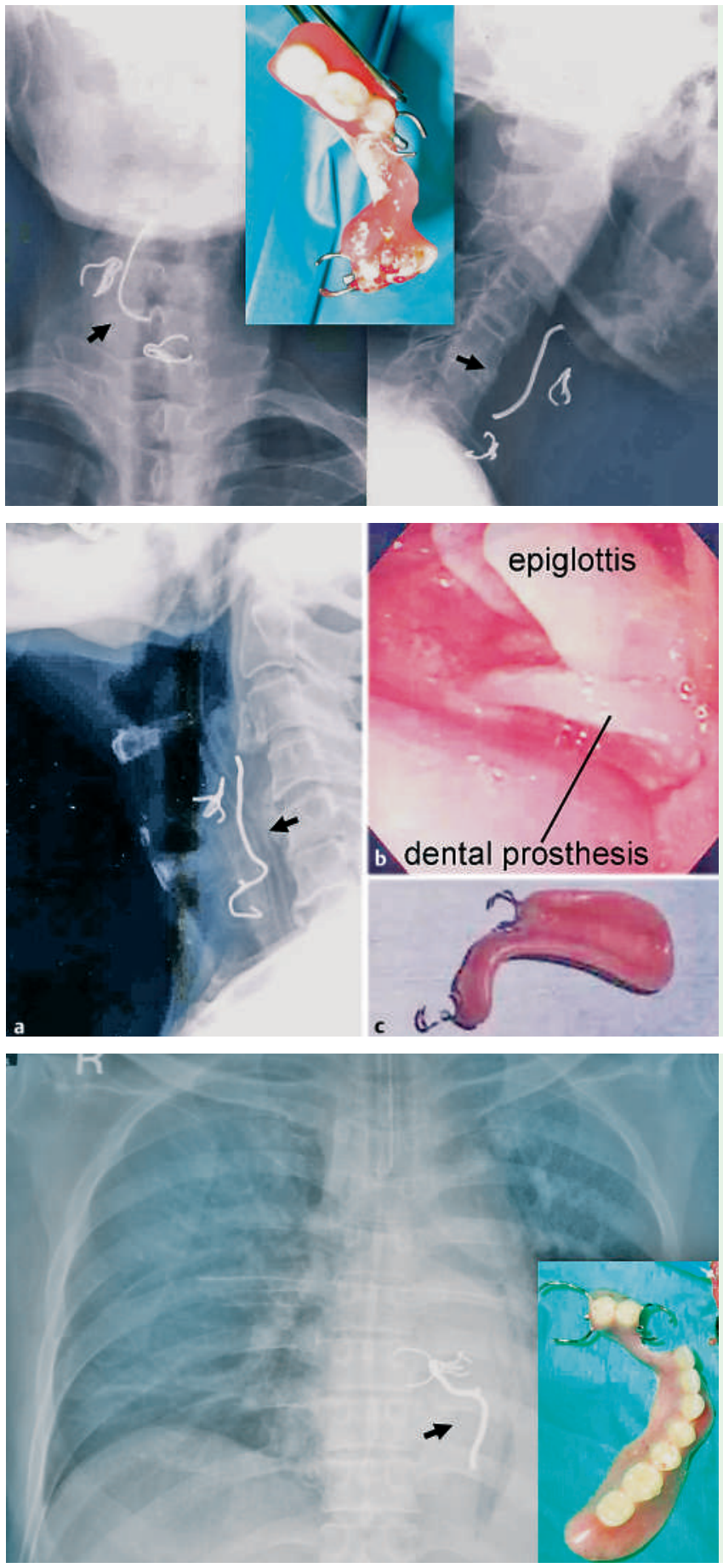

Fig. 1 Plain radiograph showing a dental prosthesis with sharp metallic clasps that are sticking into the cervical esophageal wall. Inset: the removed prosthesis.

Fig. 2 Dental prosthesis with sharp metallic clasps caught in the orifice of the esophagus, visualized by: a plain radiography, and $\mathbf{b}$ upper gastroenterological endoscopy. c The removed dental prosthesis.

Fig. 3 Plain radiograph showing dental prosthesis with sharp metallic clasps penetrating the esophageal wall. In this case the incarcerated dental prosthesis was removed with the esophagus during esophagectomy. Inset: the removed dental prosthesis.
Ingested and unremovable dental prostheses, with sharp clasps which catch and stick in the esophagus, can lead to direct injury, compression ulcer, and perforation or penetration [1,2]. To establish appropriate procedures for removing such objects according to our strategy for accidentally swallowed esophageal foreign bodies, we examined: (i) the removal process in cases without esophageal resection, and (ii) the resected specimen in a case where esophagectomy had been done.

Our strategy for the management of esophageal foreign bodies is removal as follows: (i) nonsurgically, using a direct forceps or an endoscope, by direct vertical traction or horizontal rotation, and extracting the foreign body or pushing it into the stomach; (ii) surgically, using synchronous direct manipulation under surgical exposure with the assistance of a forceps or an endoscope; or (iii) surgically, with esophagotomy or esophagectomy. Endoscopic examination is done both pre- and post-removal; in nonsurgical cases this is to check for any preceding injuries that might have been missed or for secondary injuries that occurred during removal of the foreign body, and in surgical cases it is to done to check for preceding injuries invisible from the operative field and to determine the method of reconstruction [3].

We encountered nine such cases in 6 years (involving the cervical esophagus in five cases and the thoracic in four). None of these dental prostheses with sharp clasps could be removed by simple vertical traction, but all except one could be removed by horizontal rotation ( $\bullet$ Fig. 1 and 2 ), the remaining one being removed by esophagectomy. Although post-removal endoscopy revealed ulcers in all cases, 1 or 2 days' fasting was enough to prevent perforation or penetration. In the resected specimen, we found longitudinal ulcers and perforation, indicating that the clasp had moved vertically at first, scratched the mucosa, and then rotated horizontally and penetrated the esophageal wall (๑ Fig. 3-5) [3].

Although there have been many reports concerning procedures for passing foreign bodies safely through the esophagus, there are few describing methods of extracting a dental prosthesis with sharp clasps that are sticking into the esophageal wall [3-5]. This study shows the importance of horizontal rotation for extracting such a foreign body. 


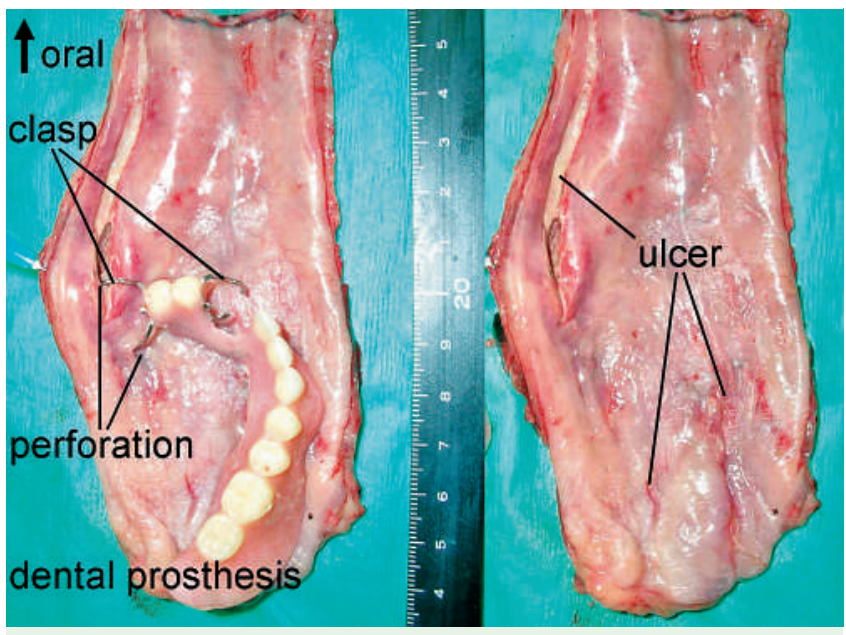

Fig. 4 Resected specimen from the patient who underwent thoracic esophagectomy. A deep longitudinal ulcer can be seen, and a perforation hole at the anal end of the ulcer in which the clasp of the dental prosthesis had been incarcerated. This indicated that the clasp of the ingested dental prosthesis had moved vertically at first, scratching the esophageal mucosa, and had then rotated horizontally and penetrated the esophageal wall, suggesting that we should attempt horizontal rotation at endoscopic removal

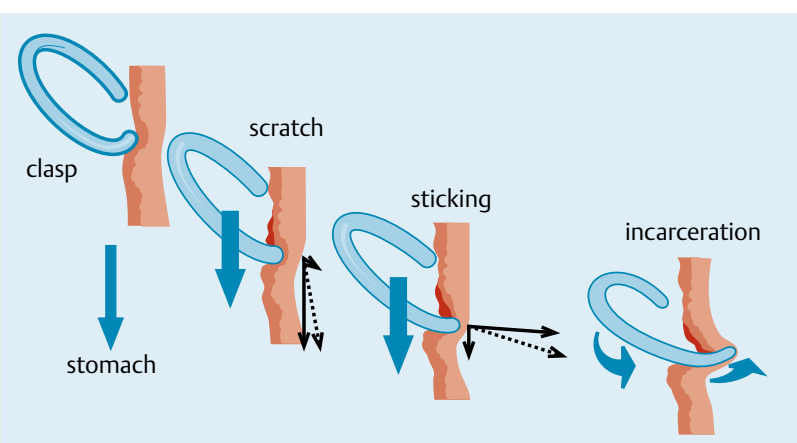

Fig. 5 Incarceration of a sharp clasp. The clasp can turn in any direction according to force (gravity or peristaltic movement) when it is not sticking into the esophageal wall. When even only the tip of the clasp has stuck, its main direction of movement is then restricted: the force that will result in further penetration into or tearing of the esophageal mucosa has a possible component along the direction of the clasp (penetration) and a possible component perpendicular to this direction (tearing), and it is difficult for the lateral surface of the shaft of the clasp to tear the layers of the esophageal wall, from the mucosal to the muscular layer, simultaneously.
Endoscopy_UCTN_Code_TTT_1AO_2AL

\section{Y. Moriwaki, M. Sugiyama, S. Arata,} H. Toyoda, T. Kosuge, N. Suzuki Critical Care and Emergency Center, Yokohama City University Medical Center, Yokohama, Japan

\section{References}

1 Abdullah BJ, Teong LK, Mahadevan J, Jalalu$\operatorname{din} A$. Dental prosthesis ingested and impacted in the esophagus and orolaryngopharynx. J Otolaryngol 1998; 27: 190-194

2 Nwaorgu OG, Onakoya PA, Sogebi OA et al. Esophageal impacted dentures. J Natl Med Assoc 2004; 96: 1350-1353

3 Moriwaki Y, Toyoda H, Kosuge T, Sugiyama $M$. A case of esophageal foreign body (dental prosthesis with sharp clasp) saved by emergency thoracic esohagectomy with simultaneous reconstruction. Jpn J Gastroenterol Surg 2006; 39: 277-282

4 Moriwaki Y, Date K, Hasegawa S, Uchida Ket al. Experience with a case of cervical esophageal foreign body (artificial tooth with clasp) in a bedridden senile. J.Jpn Surg Assoc 2002; 63: 884-889

5 Moriwaki Y, Arata S, Sugiyama M. A case of penetrating esophageal foreign body (dental prosthesis with sharp clasp) treated with endoscopic removal. Gastroenterol Endosc; In press 2007
Bibliography

DOI 10.1055/s-2007-966721

Endoscopy 2007; 39: E303-E304

() Georg Thieme Verlag KG Stuttgart · New York . ISSN 0013-726X

\section{Corresponding author \\ Y. Moriwaki, MD}

Critical Care and Emergency Center, Yokohama City University Medical Center 4-57 Urafune-cho, Minami-ku Yokohama 232-0024

Japan

Fax: +81-45-2433677

qqc3@urahp.yokohama-cu.ac.jp 